\title{
Análisis de la práctica educativa en secundaria de talleres, tecnologías y clubes a través de la historia de reformas y planes y programas
}

\section{Analysis of the educational practice in secondary workshops, technologies and clubs through the history of reforms and plans and programs}

LOPEZ-CHAVEZ, Blanca Esther†*, HERRERA-CHEW, Alejandra, SAUCEDO-SILVA, Rene y FUENTES-MORALES, María Concepción

Instituto Tecnológico de Ciudad Juárez

ID $1^{\text {er }}$ Autor: Blanca Esther, López-Chávez / ORC ID: 0000-0001-8197-9297, CVU CONACYT ID: 998072

ID $1^{\text {er }}$ Coautor: Alejandra, Herrera-Chew / ORC ID: 0000-0003-4482-4370, CVU CONACYT ID: 927452

ID $2^{\mathrm{do}}$ Coautor: Rene, Saucedo-Silva / ORC ID: 0000-0002-8123-4313, CVU CONACYT ID: 997714

ID $3^{\text {er }}$ Coautor: María Concepción, Fuentes-Morales / ORC ID: 0000-002-1400-7209, CVU CONACYT ID: 382919

DOI: $10.35429 / J E S C .2019 .10 .3 .27 .34$

Recibido: 08 de Octubre, 2019; Aceptado 26 de Noviembre, 2019

\begin{abstract}
Resumen
El propósito de este artículo es analizar los cambios en los planes y programas con el implemento de reformas educativas en el área de talleres, tecnologías y clubes desde los inicios de la educación secundaria. Se muestra cómo los talleres y tecnologías eran asignaturas que impulsaban al alumno en la preparación de un oficio. En la reforma de 2006, con la articulación de la educación básica y el planteamiento curricular de 2011, cuyo propósito en la asignatura de tecnología pretendía llevar al alumno del saber hacer a conocer los procesos necesarios para la producción de un servicio o producto, e impulsaba al alumno a ser analítico, consciente y emprendedor. La última reforma educativa de 2013, aplicada en los planes y programas de 2017 que ubica los clubes a determinar para llevarse a cabo en cuatro horas por semana. El propósito fundamental entonces de este análisis es vislumbrar el proceso de desarrollo histórico que tienen las reformas de los talleres, tecnologías y clubes en el área secundaria.
\end{abstract}

Talleres, Tecnologías, Club

\begin{abstract}
The purpose of this article is to analyze changes in plans and programs with the implementation of educational reforms in the area of workshops, technologies and clubs since the beginning of middle school. It shows how workshops and technologies were subjects that impelled the student in the preparation of a trade. In the reform of 2006, with the articulation of basic education and the curricular approach of 2011, whose purpose in the subject of technology was to take the student of know-how to know the processes necessary for the production of a service or product, and promoted the student to be analytical, conscious and enterprising. The last educational reform of 2013, applied in the plans and programs of 2017 that places the clubs to be determined to be carried out in four hours per week. The fundamental purpose then of this analysis is to glimpse the process of historical development that the reforms of the workshops, technologies and clubs in the middle school.
\end{abstract}

Citación: LOPEZ-CHAVEZ, Blanca Esther, HERRERA-CHEW, Alejandra, SAUCEDO-SILVA, Rene y FUENTESMORALES, María Concepción. Análisis de la práctica educativa en secundaria de talleres, tecnologías y clubes a través de la historia de reformas y planes y programas. Revista de Ciencias de la Educación. 2019 3-10: 27-34

\footnotetext{
* Correspondencia del Autor (correo electrónico: aherrera@itcj.edu.mx)

$\dagger$ Investigador contribuyendo como primer autor.
} 


\section{Introducción}

El presente documento analiza los cambios de la asignatura de taller, tecnología y hoy en día llamados clubes en el currículo a nivel secundaria.

De acuerdo con el proceso histórico de la implementación de los planes y programas de estudio, cada uno de ellos presenta novedades educativas cada sexenio.

Hablando específicamente de la asignatura de taller, tecnología y hoy en día club. El docente se prepara para impartir una asignatura de acuerdo a un perfil que debe cumplir, sin embargo, esta área del currículo se ha ido modificando, al inicio era más práctico que teórico, se buscaba preparar a los alumnos para un oficio.

Esta modalidad permaneció hasta la aplicación de la reforma del 2011 donde más que práctica, se buscaba que el alumno fuera más analítico, consiente y emprendedor en relación a los procesos que se llevan a cabo para la obtención de productos y servicios tanto de producción, gasto de recursos naturales y administrativos, esto de acuerdo a la tecnología cursada.

Por último, en el nuevo plan, se localiza en el tercer componente del mapa curricular, como autonomía curricular, donde se le atribuye autonomía a la comunidad escolar de elegir sus talleres, ahora nombrados clubes, de acuerdo a las necesidades, contexto y posibilidades de la misma dando la pauta a que cada alumno elija de acuerdo a sus gustos o habilidades.

\section{Metodología}

La investigación la hemos realizado a la luz de un análisis bibliográfico histórico, ya que nos interesa plasmar el desarrollo de los talleres, tecnologías y clubes en el nivel educativo de secundaria.

Nuestro enfoque principal es poner en perspectiva del lector los cambios generados por la estructuración de las diferentes reformas educativas que se han establecido en la historia del Sistema Educativo Nacional.

\section{Desarrollo}

Un antecedente remoto de la educación secundaria mexicana se encuentra en la Ley de Instrucción Pública expedida el 27 de diciembre de 1865 la cual establecía que la escuela secundaria fuese organizada al estilo del Liceo francés de acuerdo a las ideas y metodologías de Maximiliano y que su plan de estudios debería cubrirse en siete u ocho años, en los que se cursaban materias de caligrafía, conocimientos de taquigrafía, tecnología y teneduría de libros, entre otras.

Por otro lado, en la historia de la educación secundaria mexicana se reconoce al maestro Moisés Sáenz (1888-1941) como su fundador. En 1912, se incorpora como profesor de educación secundaria, lo que le permite formar sus primeras ideas sobre cómo educar a los adolescentes. En 1915 se celebró en la ciudad de Jalapa el Congreso Pedagógico de Veracruz, del cual se derivó la Ley de Educación Popular del Estado. Acontecimiento que es posible considerar como el momento en el cual se instituye y regula de manera formal la educación secundaria. Se administraba bajo el modelo tradicional; su objetivo era lograr un aprendizaje verbal y simbólico y buscaba enlazar los conocimientos experimentados en la primaria con la profesión o especialización. (MurilloGarza Angélica, 2014)

El 3 de octubre de 1921 se creó la Secretaría de Educación Pública y el 12 de octubre del mismo año, el Lic. José Vasconcelos Calderón asumió la titularidad. En materia de enseñanza técnica, Vasconcelos rechaza el pragmatismo de la escuela norteamericana ostentada por Dewey, lo que no significa rechazo al trabajo manual (la teoría curricular respondía a los intereses de la industrialización y del sistema educativo (Díaz Barriga, 2005))

Surgieron diferentes tipos de escuelas orientadas a las Escuelas de Ferrocarriles, de Industrias Textiles, Nacional de Maestros Constructores, Tecnológica para Maestros, Técnica de Artes y Oficios, Nacional de Artes Gráficas, Técnica de Taquimecanógrafos, la Escuela Hogar para Señoritas "Gabriela Mistral"; además de estas existían otras 88 de tipo técnico: mineras, industriales, comerciales y de artes y oficios, 71 de carácter oficial y 17 particulares. (SEP, s.f.). 
En 1926 se inaugura la primera escuela nocturna y se vincula a la secundaria al incluir contenidos vocacionales con las escuelas técnicas. Se crea el primer plan de estudios (1926-1939) el cual buscaba capacitar a los estudiantes para la participación en la producción y distribución de las riquezas y al mismo tiempo desarrollar su personalidad. En primer grado, el contenido curricular refleja el oficio de carpintería y/o encuadernación con tres horas a la semana y en segundo grado las temáticas generales a trabajar son: a) ¿Cómo conservar la vida? b) ¿Cómo ganarse la vida? c) ¿Cómo formar la familia? d) ¿Cómo gozar de la vida? (Murillo-Garza Angélica, 2014)

Para distinguir las secundarias de las técnicas, se modifican los programas de materias de cultura general por asignaturas de apoyo (nociones de contabilidad, costos y precios y derecho industrial).

También se crearon escuelas técnicas prevocacionales, vocacionales y de preaprendizaje en los años de 1932 y 1934 con dos años de curso cada una, las cuales impartían educación inicial en el área técnica y capacitación de un oficio a los jóvenes.

En 1934 se creó la primera escuela de pre aprendizaje con duración de dos años para la capacitación de un oficio a jóvenes, a partir de los doce años con necesidad de trabajar. Durante el segundo año los alumnos podían aspirar a un sueldo de aprendiz si el aprovechamiento era satisfactorio y además eran becados por el gobierno para poder seguir con sus estudios. (Ramírez Palacios L. R., 2012)

Durante la presidencia de Lázaro Cárdenas se impulsó la creación de escuelas vinculadas a centros de producción y se alentó la educación técnica. Así mismo la educación pública y privada fue administrada por el gobierno federal. En 1938 la SEP debido al conflicto que se generaba entre escuelas se creó un mismo plan de tres años para todas ellas (secundarias, técnicas, vocacionales, prevocacionales, entre otras), que se cursaban después de primaria, inscritas a una ideología socialista. Se buscaba una educación vocacional y técnica mediante la práctica de talleres y laboratorios para que al finalizar sus estudios se unieran al servicio comunitario para ser parte de las cooperativas del consumo y producción (Meneses, 1988).

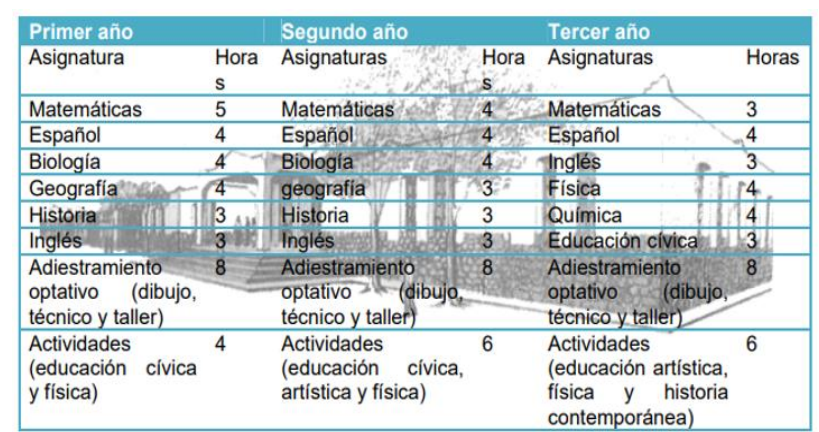

Figura 1 Plan de Estudios Prevocacionales 1939 Fuente: Ramírez Palacios L.R. 2012

Al asumir la presidencia en el contexto de la segunda guerra mundial, Manuel Ávila Camacho (1940-1946) propuso una política de unidad nacional que tuvo expresiones en la doctrina y en las políticas educativas del gobierno. Durante la posguerra, en el periodo conocido en México como de conciliación y consolidación, Miguel Alemán, sucesor de Ávila Camacho, dio continuidad a la política de industrialización y a la política educativa del gobierno anterior. El gobierno del presidente Adolfo Ruiz Cortines (1952-1958) consolidó las realizaciones educativas de los gobiernos anteriores, los servicios educativos continuaron creciendo, pero no se llevaron a cabo reformas en los métodos, programas de estudio o los textos escolares.

En 1958 con el sexenio de Adolfo López Mateos (1958-1964) entre las políticas más importantes se encuentra la formulación e inicio del Plan de Once Años, la creación e implantación de libros de texto gratuitos para la primaria, y la reforma de los planes y programas de estudio de primaria y secundaria. (OEI, Sistemas Educativos Nacionales, México)

Hasta antes de 1958 sólo existió un tipo o modalidad de educación secundaria, a la que posteriormente se le denominó general para distinguirla de la que se denomina secundaria técnica; esta última, además de ofrecer una educación en ciencias y humanidades, incluyó actividades tecnológicas para promover en el educando una preparación para el trabajo. (Zorrilla, 2004)

Durante el gobierno de Díaz Ordaz (1964-1970) se creó una escuela técnica por cada secundaria general y se elaboró un mismo plan y programa de estudios para ambas y se impulsaron las escuelas secundarias técnicas agropecuarias. 
Durante el gobierno de Luis Echeverría (1970-1976) se propició la reforma a los planes y programas de primaria y secundaria. Se acordó que la secundaria al igual que la primaria se organizara por áreas de conocimiento en vez de asignaturas, dichas áreas fueron: matemáticas, español, ciencias naturales -que agrupaba biología, física y química-, ciencias sociales -historia, civismo y geografía-, y además estaban las materias de tecnología, educación física y educación artística. La reforma, como se circunscribió al tema curricular y pedagógico, estuvo vigente hasta principios de los años noventa. (Zorrilla, 2004), misma que no se logró implantar en todas las escuelas y en 1974 la SEP autoriza a las escuelas secundarias optar por los nuevos programas de áreas o por los anteriores de asignaturas.

El gobierno de López Portillo (19761982) elaboró el Plan Nacional de Educación (PNE) el cuál consistió en un diagnóstico y en un conjunto de programas y objetivos. En 1978 se declaró prioritaria la educación preescolar. También en este periodo se creó la Universidad Pedagógica Nacional (UPN).

La presidencia de Miguel de la Madrid, (1982-1988), inició en el momento en que se desencadenó una fuerte crisis económica. La educación secundaria perdió dinamismo hasta llegar al estancamiento en 1988. Esto obedeció a la reducción de la cantidad de egresados de primaria y a que un número cada vez mayor de éstos interrumpió sus estudios.20 (OEI).

El 18 de mayo de 1992 la federación, los gobiernos estatales y el Sindicato Nacional de Trabajadores de la Educación firmaron el Acuerdo Nacional para la Modernización de la Educación Básica (ANMEB), con el propósito de dar solución a las desigualdades educativas nacionales y mejorar la calidad de la educación básica a través de tres estrategias fundamentales de política educativa, por las que se conoce como el Acuerdo de las tres "erres":

a) La reorganización del sistema educativo, lo que se conoce como federalización descentralizadora; a través de este proceso, el gobierno federal transfirió a los 31 estados del país los recursos y la responsabilidad de operar sus sistemas de educación básica (preescolar, primaria y secundaria). b) La reformulación de contenidos y materiales educativos implicó una reforma curricular y pedagógica. Se renuevan contenidos como antes de la reforma de 1973, por asignaturas, se amplía y diversifica la producción de materiales educativos para alumnos y maestros.

c) Revaloración social de la función magisterial.

\begin{tabular}{|c|c|c|}
\hline \multicolumn{3}{|c|}{ Asignaturas académic } \\
\hline Español & Español & Español \\
\hline $5 \mathrm{~h}$ semanales & $5 \mathrm{~h}$ semanales & $5 \mathrm{~h}$ semanales \\
\hline Matemáticas & Matemáticas & Matemáticas \\
\hline $5 \mathrm{~h}$ semanales & $5 \mathrm{~h}$ semanales & $5 \mathrm{~h}$ semanales \\
\hline $\begin{array}{l}\text { Historia } \\
\text { Universal I }\end{array}$ & $\begin{array}{l}\text { Historia } \\
\text { Universal II }\end{array}$ & $\begin{array}{l}\text { Historia de } \\
\text { México }\end{array}$ \\
\hline $3 \mathrm{~h}$ semanales & $3 \mathrm{~h}$ semanales & $3 \mathrm{~h}$ semanales \\
\hline Geografía general & $\begin{array}{l}\text { Geografía de } \\
\text { México }\end{array}$ & $\begin{array}{l}\text { Orientación } \\
\text { educativa }\end{array}$ \\
\hline $3 \mathrm{~h}$ semanales & $2 \mathrm{~h}$ semanales & $3 \mathrm{~h}$ semanales \\
\hline Civismo & Civismo & Civismo \\
\hline $3 \mathrm{~h}$ semanales & $3 \mathrm{~h}$ semanales & $3 \mathrm{~h}$ semanales \\
\hline Biología & Biología & Biología \\
\hline $3 \mathrm{~h}$ semanales & $3 \mathrm{~h}$ semanales & $3 \mathrm{~h}$ semanales \\
\hline $\begin{array}{l}\text { Introducción a la } \\
\text { física y a la Química }\end{array}$ & Física & $\begin{array}{l}\text { Lengua } \\
\text { extranjera }\end{array}$ \\
\hline $3 \mathrm{~h}$ semanales & $3 \mathrm{~h}$ semanales & $3 \mathrm{~h}$ semanales \\
\hline Lengua extranjera & $\begin{array}{l}\text { Lengua } \\
\text { extranjera }\end{array}$ & $\begin{array}{l}\text { Asignatura } \\
\text { opcional }\end{array}$ \\
\hline $3 \mathrm{~h}$ semanales & $3 \mathrm{~h}$ semanales & $3 \mathrm{~h}$ semanales \\
\hline
\end{tabular}

\begin{tabular}{|l|l|l|}
\hline \multicolumn{2}{|l|}{ Desarrollo de actividades } \\
\hline Primero & Segundo & Tercero \\
\hline $\begin{array}{l}\text { Expresión y } \\
\text { apreciación } \\
\text { artística }\end{array}$ & $\begin{array}{l}\text { Expresión y } \\
\text { apreciación } \\
\text { artística }\end{array}$ & $\begin{array}{l}\text { Expresión y } \\
\text { apreciación } \\
\text { artística }\end{array}$ \\
\hline 2 h semanales & 2 h semanales & 2 h semanales \\
\hline $\begin{array}{l}\text { Educación } \\
\text { física }\end{array}$ & $\begin{array}{l}\text { Educación } \\
\text { física }\end{array}$ & $\begin{array}{l}\text { Educación } \\
\text { física }\end{array}$ \\
\hline 2 h semanales & 2 h semanales & 2 h semanales \\
\hline $\begin{array}{l}\text { Educación } \\
\text { tecnológica }\end{array}$ & $\begin{array}{l}\text { Educación } \\
\text { tecnológica }\end{array}$ & $\begin{array}{l}\text { Educación } \\
\text { tecnológica }\end{array}$ \\
\hline 3 h semanales & $3 \mathrm{~h}$ semanales & $3 \mathrm{~h}$ semanales \\
\hline $\begin{array}{l}\text { Totales: } 35 \mathrm{~h} \\
\text { semanales }\end{array}$ & $\begin{array}{l}\text { Totales: } 35 \mathrm{~h} \\
\text { semanales }\end{array}$ & $\begin{array}{l}\text { Totales: } 35 \mathrm{~h} \\
\text { semanales }\end{array}$ \\
\hline
\end{tabular}

Tabla 1 Contenidos por Asignatura

Una consecuencia del ANMEB fue el establecimiento, en julio de 1993, de la obligatoriedad de la educación secundaria incrementándose a 9 años la escolaridad básica obligatoria; es decir, seis de primaria y tres de secundaria.

Esta decisión implicó una reforma importante del artículo tercero de la Constitución. 
Para ser profesor de secundaria había que cursar lo que se denominaba "la normal superior". La normal superior tenía grado de licenciatura y se organizaba por especialidades de materias según el currículo de la enseñanza secundaria.

Persisten tres modalidades de atención, la secundaria general cuyo modelo se parece más al del bachillerato, la secundaria técnica que conserva la enseñanza de tecnologías específicas que le permitan al alumno eventualmente incorporarse al mercado de trabajo y la telesecundaria. (Zorrilla, La Educación Secundaria En México: Al filo de su reforma, 2004)

El Programa Nacional de Educación 2001-2006 establece como uno de sus objetivos prioritarios la reforma integral de la educación secundaria. La meta para el año 2004 es la de contar con una propuesta de renovación curricular, pedagógica y organizativa de la educación secundaria, incluidos la revisión y el fortalecimiento del modelo de atención de la telesecundaria.

Se busca romper con el enfoque academicista que ha tenido desde sus orígenes. Así, el diseño del plan y programas de estudio tiene el desafío de articular de nuevas maneras un enfoque basado en competencias generales como son la comprensión lectora, las habilidades matemáticas y la transmisión de valores éticos y ciudadanos.

El 26 de mayo de 2006, mediante el Acuerdo Secretarial 384, se hizo oficial el nuevo Plan y programas de estudio para la educación secundaria para que diera inicio en el ciclo escolar 2006-2007 en todo el país. (Mirnada López \& Reynoso Angulo, 2006)

\begin{tabular}{|c|c|c|c|c|c|}
\hline Primer grado & Horas & Segundo grado & Horas & Tercer grado & Horas \\
\hline Español I & 5 & Español II & 5 & Español III & 5 \\
\hline Matemáticas I & 5 & Matemáticas II & 5 & Matemáticas III & 5 \\
\hline $\begin{array}{c}\text { Ciencias I } \\
\text { (énfasis en Biología) }\end{array}$ & 6 & $\begin{array}{c}\text { Ciencias II } \\
\text { (énfasis en Física) }\end{array}$ & 6 & $\begin{array}{c}\text { Ciencias III } \\
\text { (énfasis en Química) }\end{array}$ & 6 \\
\hline \multirow[t]{2}{*}{$\begin{array}{c}\text { Geografía de } \\
\text { México y del Mundo } \\
\end{array}$} & 5 & Historia I & 4 & Historia II & 4 \\
\hline & & $\begin{array}{c}\text { Formación Cívica } \\
\text { YÉtical } \\
\end{array}$ & 4 & $\begin{array}{c}\text { Formación Cívica } \\
\text { YÉtica II } \\
\end{array}$ & 4 \\
\hline Lengua Extranjera I & 3 & Lengua Extranjera II & 3 & Lengua Extranjera III & 3 \\
\hline $\begin{array}{c}\text { Educación } \\
\text { Física I }\end{array}$ & 2 & $\begin{array}{c}\text { Educación } \\
\text { Física II }\end{array}$ & 2 & $\begin{array}{c}\text { Educación } \\
\text { Física III }\end{array}$ & 2 \\
\hline Tecnología I & 3 & Tecnología II & 3 & Tecnología III & 3 \\
\hline $\begin{array}{c}\text { Artes (Música, Danza, } \\
\text { Teatro o Artes } \\
\text { Visuales) } \\
\end{array}$ & 2 & $\begin{array}{c}\text { Artes (Música, Danza, } \\
\text { Teatro o Artes } \\
\text { Visuales) } \\
\end{array}$ & 2 & $\begin{array}{c}\text { Artes (Música, Danza, } \\
\text { Teatro o Artes } \\
\text { Visuales) } \\
\end{array}$ & 2 \\
\hline Asignatura Estatal & 3 & & & & \\
\hline Orientación y Tutoría & 1 & Orientación y Tutoría & 1 & Orientación y Tutoría & 1 \\
\hline Total & 35 & & 35 & & 35 \\
\hline
\end{tabular}

Tabla 2 Programa de Estudio para Educación Secundaria a partir del año 2006

ISSN: 2523-2436

ECORFAN® Todos los derechos reservados
En la administración del Presidente Felipe Calderón (2006-2012) se puso en marcha un proceso de modernización llamada La Alianza por la Calidad de la Educación, luego de coincidir en que el modelo educativo ya no respondía a las exigencias del mundo moderno, debido a que fue creado en el siglo pasado. (SEP, 2008)

La Alianza por la Calidad de la Educación, suscrita el 15 de mayo del 2008 entre el Gobierno Federal y los maestros de México representados por el Sindicato Nacional de Trabajadores de la Educación (SNTE), estableció el compromiso de llevar a cabo una reforma curricular orientada al desarrollo de competencias y habilidades, mediante la reforma a los enfoques, asignaturas y contenidos de la Educación Básica y la enseñanza del idioma inglés desde el nivel preescolar.

Más allá de lo que ocurre en el aula, la Alianza por la Calidad de la Educación también generó compromisos encaminados a modernizar los centros escolares con el fin de fortalecer su infraestructura y modernizar el equipamiento de los planteles escolares para conectarlos a redes de alto desempeño, así como ampliar su gestión y participación social en la determinación y el seguimiento de los proyectos estratégicos de transformación escolar; visión que fortaleció a la Reforma Integral de la Educación Básica.

En consecuencia, el 19 de agosto del 2011 se publica en el Diario Oficial de la Federación el acuerdo 592 donde se establece la articulación de la educación básica organizada en un Plan y programas de estudio.

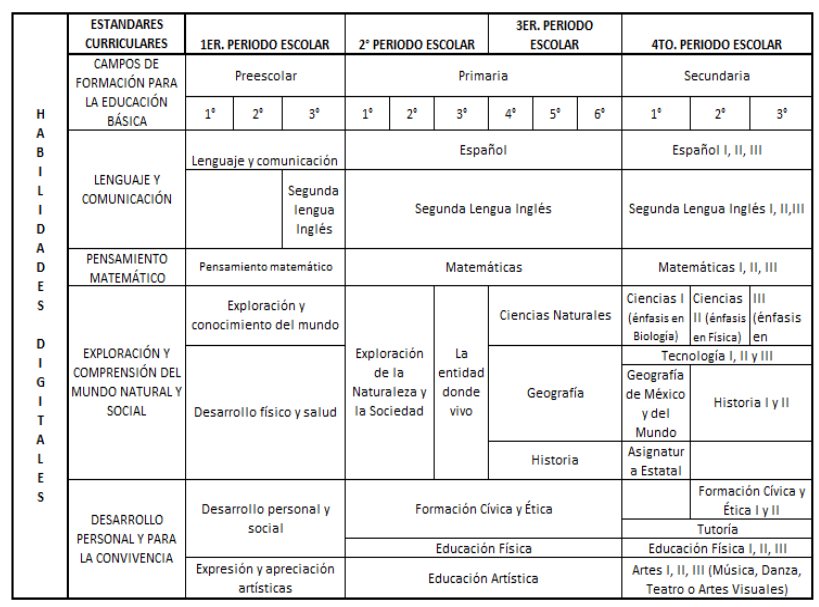

Tabla 3 Mapa Curricular de la Educación Básica 2011 
El 20 de diciembre del 2012 se aprueba por la Cámara de Diputados y por el Senado de la República, reforma constitucional en materia educativa, presentada por el Presidente Enrique Peña Nieto. Promulgada el 26 de febrero de 2013 (DOF: 26/02/2013) La cual otorga al Sistema Educativo Nacional de los elementos que impulsen su mejoramiento y fortalezcan la equidad. Asegura la obligación del Estado de garantizar la calidad de la educación pública obligatoria; la creación de un servicio profesional docente; el establecimiento del Sistema Nacional de Evaluación Educativa, y la constitución del Instituto Nacional para la Evaluación de la Educación, como máxima autoridad en materia de evaluación.

El 29 de junio de 2017 el DOF establece los planes y programas para la educación básica llamados Aprendizajes Clave para la educación integración.

Un aprendizaje clave es un conjunto de conocimientos, prácticas, habilidades, actitudes $\mathrm{y}$ valores fundamentales que contribuyen sustancialmente al crecimiento integral del estudiante

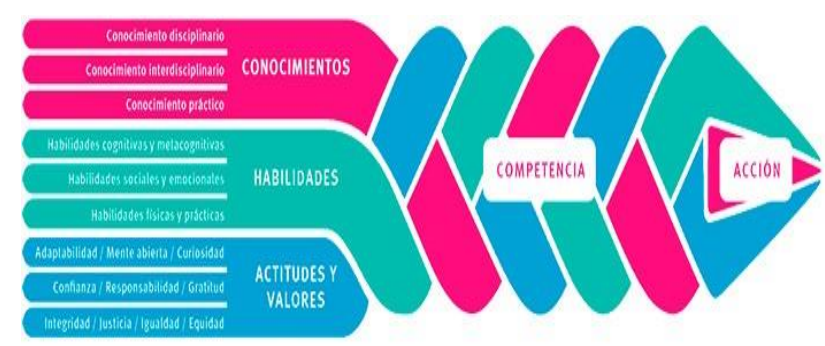

Figura 2 Aprendizajes Clave

Fuente: DOF, Acuerdo número 07/06/17

Cada componente del nuevo modelo incluye sus espacios curriculares específicos: asignaturas, en el caso del componente Formación académica; Áreas de Desarrollo, en el caso del componente Desarrollo personal y social; y ámbitos, en el caso del componente Autonomía curricular.

El tercer componente se rige por los principios de la educación inclusiva porque busca atender las necesidades educativas e intereses específicos de cada educando. Cada escuela elegirá e implementará la oferta de este componente curricular con base en los periodos lectivos que tenga disponibles y en los lineamientos que expida la SEP.
El componente está organizado en cinco ámbitos: "Ampliar la formación académica", "Potenciar el desarrollo personal y social", "Nuevos contenidos relevantes", "Conocimientos regionales" y "Proyectos de impacto social". (DOF, Acuerdo número 07/06/17 por el que se establece el Plan y los Programas de Estudio para la Educación Básica: Aprendizajes clave para la educación integral., 2017)

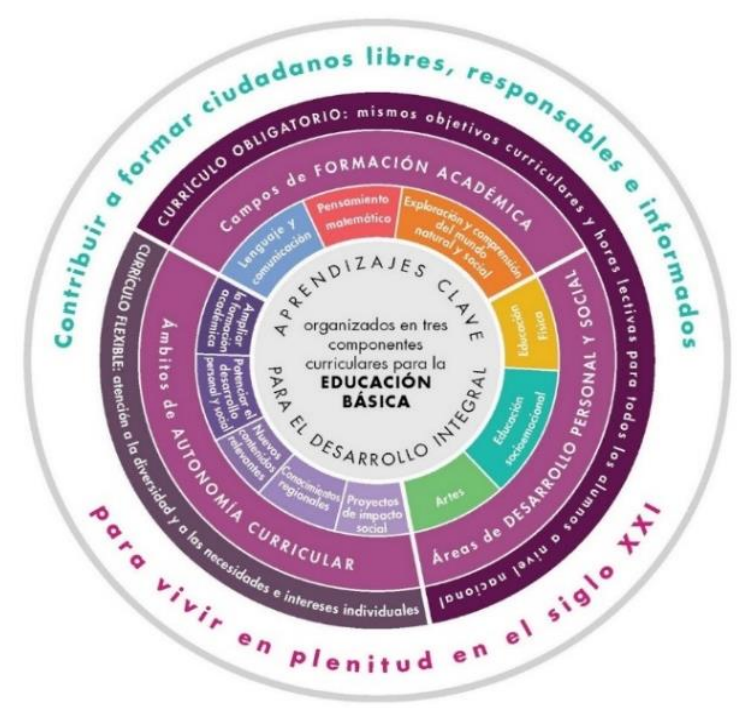

Figura 3 Aprendizajes Clave para la Educación Integral Fuente: DOF, Acuerdo número 07/06/17

\section{Resultados}

En un inicio los talleres se clasificaban en el área de adiestramiento con 8 horas semanales por grado, después su término cambio a educación tecnológica y se encontraba en el currículo especificada en el desarrollo de una actividad, con una disminución a 3 horas. En la actualidad, con el Nuevo Modelo Educativo "Aprendizajes Clave" se ubica en el apartado de autonomía curricular, aumentando de 3 a 4 horas semanales.

Particularmente, hablando en esta área del mapa curricular de secundaria, se reflejan cambios de acuerdo a necesidades que se señalan en las distintas reformas educativas implementadas, las cuales reflejan la preocupación de mejorar la educación, basados en el tipo de sociedad, lo que se requiere y se necesita formar. Con ello las reformas se basan para cambiar e implementar nuevos planes y programas con el que el docente, debe guiarse y guiar a través de la práctica diaria con sus estudiantes, buscando generar ambientes de aprendizaje dentro del aula. 
Es de reconocerse los objetivos planteados e impuestos por las reformas y en consecuencia los cambios del currículo, procedimientos que mencionan entre algunos apartados, la profesionalización y capacitación del docente. Un docente frente a grupo es el responsable de generar conocimiento a través del proceso enseñanza-aprendizaje a sus alumnos, este a su vez debe estar sustentado con una planeación para justificar lo realizado dentro del aula. La contribución de esta investigación está orientada a que el lector visualice los cambios generados a través de las reformas educativas subyacentes en nuestro Sistema Educativo Nacional en materia de talleres, tecnología y clubes en el periodo de tiempo ya mencionado anteriormente.

\section{Conclusión}

De acuerdo a la bibliografía consultada, la educación secundaria se encuentra en la modalidad de general, técnica y telesecundaria, las cuales se fueron creando de acuerdo a las necesidades planteadas en cada sexenio, mismas que en cada una de las reformas elaboradas son justificadas y generan propuestas de cambio en el currículo que se presenta en los planes de estudio. De acuerdo al contexto nacional del momento, como un antecedente a los talleres y tecnologías en el nivel educativo de secundaria, existían escuelas que se cursaban después de la primaria, además de la secundaria, con el objetivo de formar estudiantes para el trabajo, su finalidad era la capación en un oficio, particularidad que cambio. Por cada escuela secundaria general se creó una técnica en las cuales se llevaba un mismo plan de estudios vigente hasta los años noventa, su distinción era que en la técnica al educando se daba una preparación para el trabajo con más horas a la semana, a diferencia de la general que solo se impartía tres.

Fue hasta el siglo XXI con la reforma del 2006, cuando se pensó en crear alumnos analíticos y críticos de los procesos de cada producto o servicio que ellos mismos experimentan. Surge la necesidad de crear conciencia bajo el sustento de la ciencia, crear alumnos con aspiraciones de ser el dueño de su propia empresa. Presentado en el plan y programas del 2011 con el nombre de tecnologías, 3 horas semanales para las secundarias generales y para las técnicas se aumentaba según su funcionamiento y objetivo.
Hoy en día, se generaliza en las secundarias el tiempo destinado al área de los clubs a cuatro horas semanales. Dentro del currículo se clasifica en el componente de autonomía |curricular, mismos que a través del desarrollo histórico del país ha sido impartida y experimentada generalmente por los docentes que antes impartieron la asignatura de tecnología.

\section{Referencias}

Díaz Barriga, Á. (2005). El docente y los programas escolares Lo institucional y lo didáctico (Ediciones Pomares, S. A. ed.). México. Recuperado el 25 de 01 de 2019

DOF. (2001). Acuerdo 592 por el que se establece la Articulación de la Educación Básica. Obtenido de https://www.sep.gob.mx/work/models/sep1/Res ource/9721849d-666e-48b7-8433-

0eec 1247f1ab/a592.pdf

DOF. (07 de 06 de 2017). ACUERDO número 07/06/17 por el que se establece el Plan y los Programas de Estudio para la Educación Básica: Aprendizajes clave para la educación integral. Obtenido de SEGOB (Secretaría de Gobernación):

https://www.dof.gob.mx/nota_detalle.php?codi go $=5488475 \&$ fecha $=29 / 06 / 2017$

Gutierrez G., J. A. (s.f.). Ley de instrucción pública de Maximiliano. Recuperado el 15 de 01 de 2019, de https://archivos.juridicas.unam.mx/www/bjv/lib ros/10/4551/7.pdf

INEE. (2015). Reforma Educativa, Marco Normativo. Obtenido de http://www.senado.gob.mx/comisiones/educaci on/docs/docs_INEE/Reforma_Educativa_Marc o_normativo.pdf

La Educación Secundaría en México: Al filo de su reforma. (2004). REICE - Revista Electrónica Iberoamericana sobre Calidad, Eficacia $y$ Cambio en Educación, 2(1), 4. Recuperado el 15 de 1 de 2019, de https://www.redalyc.org/html/551/55120106/ México, G. d. (s.f.). Reforma Educativa (Resumen ejecutivo). Obtenido de https://www.gob.mx/cms/uploads/attachment/fi le/2924/Resumen_Ejecutivo_de_la_Reforma_E ducativa.pdf

LOPEZ-CHAVEZ, Blanca Esther, HERRERA-CHEW, Alejandra, SAUCEDO-SILVA, Rene y FUENTES-MORALES, María Concepción. Análisis de la práctica educativa en secundaria de talleres, tecnologías y clubes a través de la historia de reformas y planes y programas. Revista de Ciencias de la Educación. 2019 
Mirnada López, F., \& Reynoso Angulo, R. (oct.dic. de 2006). La Reforma de la Educación Secundaria en México. Elementos para el debate. Revista Mexicana de Investigación Científica, 11(31), 1427-1450 (P.16). Recuperado el 29 de 01 de 2019, de https://www.redalyc.org/pdf/140/14003115.pdf

Murillo-Garza Angélica, M.-P. J.-S.-L. (2014). Moisés Sáenz Garza, Transformador de la Realidad. Revista Iberoamericana de Ciencias, 5. Recuperado el 15 de 01 de 2019, de http://www.reibci.org/publicados/2014/septiem bre/3300124.pdf

OEI. (s.f.). Sistemas educativos nacionales, México. Recuperado el 28 de 01 de 2019, de Evolución del sistema educativo: file://localhost/C:/Users/SONY/Downloads/me x02 (3).pdf

OEI. (s.f.). Sistemas Educativos Nacionales, México. Recuperado el 28 de 01 de 2019, de Evolución del sistema educativo mexicano: file://localhost/C:/Users/SONY/Downloads/me x02 (3).pdf

OEI, O. d. (s.f.). Sistemas Educativos Nacionales. Recuperado el 28 de 01 de 2019, de Evolución del Sistema Educativo Mexicano: file://localhost/C:/Users/SONY/Downloads/me x02 (3).pdf

Quiroz, R. (1998). La reforma de 1993 de la educación secundaria en México: nuevo currículum y prácticas de esnseñanza. Imvestigación en la Escuela, 89. Recuperado el 29 de 01 de 2019, de http://www.investigacionenlaescuela.es/articulo s/36/R36_5.pdf

Ramírez Palacios, L. (19 de 06 de 2012). Repositorio Digital IPN. Recuperado el 25 de 01 de 2019, de Las escuelas prevocacionales en el IPN,

1936-1947:

https://www.repositoriodigital.ipn.mx/handle/1 $23456789 / 5576$

Ramírez Palacios, L. R. (19 de 06 de 2012). Repositorio digital IPN. Recuperado el 18 de 1 de 2019, de Las escuelas prevocacionales en el IPN, 1936-1947: https://www.repositoriodigital.ipn.mx/handle/1 23456789/5576
Rogelio, A. A. (s.f.). Reformas educativas en secundaria. Recuperado el 15 de 01 de 2019, de Historia de la educación secundaria: $\mathrm{http} / / /$ reformaseducativasensecundaria.blogspot .com/2011/03/historia-de-la-educacionsecundaria-en.html

SEP. (s.f.). Obtenido de Creación de la Secretaría de Educación Pública: https://www.gob.mx/sep/acciones-yprogramas/historia-de-la-secretaria-deeducacion-publica-15650? state $=$ published

SEP. (16 de mayo de 2008). Presenta el gobierno federal alianza estratégica para la transformar la política educativa. Obtenido de http://www.sems.gob.mx/en_mx/sems/Port_16 052008_presenta_el_gobierno_federal_alianza

SEP. (2013). Programas de estudio 2011. México.

Zorrilla, M. (2004). La educación secundaria en México: Al filo de se reforma. REICE - Revista Electrónica Iberoamericanasobre Calidad, Eficacia y Cambio en Educación, 2(1), 6. Recuperado el 28 de 01 de 2019, de file://localhost/C:/Users/SONY/Downloads/edu cacion_secundaria_mexico_filo_reforma_zorril la (2).pdf

Zorrilla, M. (2004). La Educación Secundaria en México: Al filo de su reforma. REICE - Revista Electrónica Iberoamericanasobre Calidad, Eficacia y Cambio en Educación, 2(1), 7. Recuperado el 29 de 1 de 2019, de file://localhost/C:/Users/SONY/Downloads/edu cacion_secundaria_mexico_filo_reforma_zorril la (2).pdf

Zorrilla, M. (2004). La Educación Secundaria en México: Al filo de su reforma. REICE - Revista Electrónica Iberoamericanasobre Calidad, Eficacia y Cambio en Educación, 2(1), 16, 2021. Recuperado el 29 de 01 de 2019, de file://localhost/C:/Users/SONY/Downloads/edu cacion_secundaria_mexico_filo_reforma_zorril la (2).pdf

Zorrilla, M. (2004). La Educación Secundaria En México: Al filo de su reforma. REICE Revista Electrónica Iberoamericanasobre Calidad, Eficacia y Cambio en Educación, 2(1), 8. Recuperado el 29 de 01 de 2019, de file://localhost/C:/Users/SONY/Downloads/edu cacion_s

LOPEZ-CHAVEZ, Blanca Esther, HERRERA-CHEW, Alejandra, SAUCEDO-SILVA, Rene y FUENTES-MORALES, María Concepción. Análisis de la práctica educativa en secundaria de talleres, tecnologías y clubes a través de la historia de reformas y planes y programas. Revista de Ciencias de la Educación. 2019 